\title{
The effect of artificial diffusivity on the flute instability
}

\author{
D. D. Ryutov, ${ }^{\text {a) }}$ B. I Cohen, R. H. Cohen, and E. B. Hooper \\ Lawrence Livermore National Laboratory, Livermore, California 94551 \\ C. R. Sovinec \\ Department of Engineering Physics, University of Wisconsin, Madison, Wisconsin 53706
}

(Received 20 May 2005; accepted 18 July 2005; published online 19 August 2005)

\begin{abstract}
Sometimes, in order to improve the performance of magnetohydrodynamical codes, artificial diffusivity $(D)$ is introduced in the mass continuity equation. In this communication, an analysis of the effect of the artificial diffusivity on the low- $\beta$ plasma stability in a simple geometry is presented. It is shown that, at low diffusivity, one recovers classical results, whereas at high diffusivity the plasma becomes more unstable. Dependence of the stability on $D$ is suppressed if the volume of the flux tube varies insignificantly in the course of the perturbation growth. These observations may help the code developers and users to identify the regimes where the artificial diffusivity is not affecting the results (or vice versa). (C) 2005 American Institute of Physics.
\end{abstract}

[DOI: $10.1063 / 1.2030367$ ]

When performing magnetohydrodynamic (MHD) numerical simulations of the plasma dynamics, one sometimes adds artificial diffusivity to the mass continuity equation (see, e.g., Refs. 1-4). This allows one to eliminate formation of too steep density gradients and, most importantly, of voids, whose appearance might make a code crash. Another reason for adding artificial diffusivity is to suppress numerical noise in nonlinear calculations. In fact, many numerical algorithms introduce numerical dissipation for this purpose, effectively adding a hidden diffusivity that ultimately decreases with increasing resolution but varies in time and space. On the other hand, the presence of this "smoothing" term may affect the results of the simulations and introduce some unphysical effects. In order to address these concerns, we present here an analytic solution of one of the most generic MHD problems, that of the flute stability, ${ }^{5}$ with the continuity equation containing an artificial diffusivity. The solution presented provides some insights on the possible impact of artificial diffusivity and allows one to identify the situations where this impact may be substantial. In some cases, the results are rather surprising: We find instability in the regimes where neither ideal nor dissipative instability would be normally expected. We relate this to a peculiar effect that artificial diffusivity has on the energy conservation [see Eq. (2) below].

We are not aiming at explaining any particular set of simulations for any particular device, but rather provide a general discussion that may serve as guidance for considering specific cases and identify regimes where the effect of artificial diffusivity is negligible or small. We are not aware of any published analyses of this kind.

The set of MHD equations used in this communication are as follows:

$$
\rho\left\lfloor\frac{\partial \mathbf{v}}{\partial t}+(\mathbf{v} \cdot \nabla) \mathbf{v}\right\rfloor=-\nabla p+\frac{1}{c} \boldsymbol{j} \times \boldsymbol{B},
$$

\footnotetext{
${ }^{a)}$ Author to whom correspondence should be addressed. Electronic mail: ryutov1@1lnl.gov
}

$$
\begin{aligned}
& \frac{\partial \rho}{\partial t}+\nabla \cdot(\rho \mathbf{v})=D \nabla^{2} \rho, \\
& \frac{3}{2} \rho \frac{\partial T}{\partial t}+\frac{3}{2} \rho \mathbf{v} \cdot \nabla T+\rho T \nabla \cdot \mathbf{v}=0, \\
& \frac{\partial \boldsymbol{B}}{\partial t}=\nabla \times[\mathbf{v} \times \boldsymbol{B}], \\
& \nabla \times \boldsymbol{B}=\frac{4 \pi}{c} \boldsymbol{j}, \\
& p=2 \rho T / m_{i},
\end{aligned}
$$

where $\rho$ is the mass density, $\mathbf{v}$ is the plasma velocity, $T$ is the temperature (common for the electrons and ions), $\boldsymbol{B}$ is the magnetic field, $\boldsymbol{j}$ is the current density, $p$ is the pressure, $D$ is the artificial diffusivity coefficient, and $m_{i}$ is the ion mass. The cgs (Gaussian) system of units is used.

Aside from the presence of the artificial diffusivity $D$ in the continuity equation, these are equations of ideal MHD. We have dropped all the dissipative processes (except for the artificial diffusivity) in order to more clearly isolate the effects of the finite $D$. Later on in this communication we will also discuss the role of viscous effects, because an artificial viscosity is also sometimes used in the simulations; we also discuss resistive effects.

The concerns about the effect of the artificial diffusivity are related to the fact that it is not easy to provide a physical picture that would lead to the appearance of the diffusion term in the continuity equation. One can of course take a viewpoint that it is just a specific model of a particle source, a model for which the source is proportional to $\nabla^{2} \rho$. However, aside from the somewhat artificial structure of this source, one would then also have to explain why the presence of this source in the continuity equation is not accompanied by the appearance of some sources in the momentum equation Eq. (1a) and temperature equation Eq. (1c). Making 
the sources and sinks of the momentum and particle energy such that the corresponding terms would not show up in either of the aforementioned equations is possible, but it is difficult to associate them with any real sources. (The particles would have to be thrown in or taken out at the velocity equal to the velocity of the ambient plasma, and with the temperature equal to the temperature of the ambient plasma, at a rate proportional to $\nabla^{2} \rho$.) All this is reflected in the fact that the energy integral for the set (1),

$$
\begin{aligned}
& \frac{d}{d t} \int_{V}\left(\frac{3}{2} p+\frac{\rho v^{2}}{2}+\frac{B^{2}}{8 \pi}\right) d V \\
& =\int_{V} D\left(\nabla^{2} \rho\right)\left(\frac{v^{2}}{2}+\frac{3 T}{m_{i}}\right) d V \\
& =-\int_{V} D(\nabla \rho) \cdot \nabla\left(\frac{v^{2}}{2}+\frac{3 T}{m_{i}}\right) d V,
\end{aligned}
$$

contains a non-sign-definite term proportional to $D$. Just this is what makes difficult any a priori judgment of its possible role in the stability. [The energy integral (2) is written for the plasma surrounded by impermeable, perfectly conducting walls.]

Artificial diffusivity should not be confused with the plasma diffusion caused by a finite resistivity, sometimes anomalous. This latter effect, if present, enters the problem via Eq. (1d), in the right-hand side (RHS) of which one then has to add a term $-(1 / c) \nabla \times(j / \sigma)$, where $\sigma$ is the electrical conductivity. This change shows up in the energy conservation law, Eq. (2), where a sign-definite and easy-to-interpret term $-j^{2} / \sigma$ appears in the integrand of the RHS. The resistive diffusion sets in because of the Joule dissipation of a diamagnetic current and is perfectly consistent with the standard energy conservation law. In the problem under consideration, the resistive term just slows down the growth of flute perturbations. We comment more on this issue later in the paper.

The aforementioned problems do not mean that the use of the artificial diffusivity would necessarily lead to substantial qualitative errors in the description of the plasma behavior. One can expect that in problems where the plasma compressibility is unimportant, the effect of a very large $D$ will be weak. Our results support this viewpoint.

Given the lack of a clear physical meaning of the artificial diffusivity (its presence implies that a kind of the "Maxwell demon" acts in a plasma, throwing the particles in or out by a very peculiar set of rules), we have taken the following approach. We just apply the set (1) to the analysis of a well-known plasma physics problem, that of the flute instability in a simple geometry, and see what happens. If the solution found does not deviate strongly from the "standard" solution, this signifies that the diffusion term is harmless.

The geometry is illustrated by Fig. 1(a). A cylindrically symmetric plasma, uniform in the axial direction, is immersed in a purely azimuthal magnetic field of the current rod. We consider a low- $\beta$ plasma so that $\beta \equiv 8 \pi p / B^{2} \ll 1$. In the equilibrium, we have a resting plasma with a uniform density (therefore satisfying the unperturbed continuity equa-

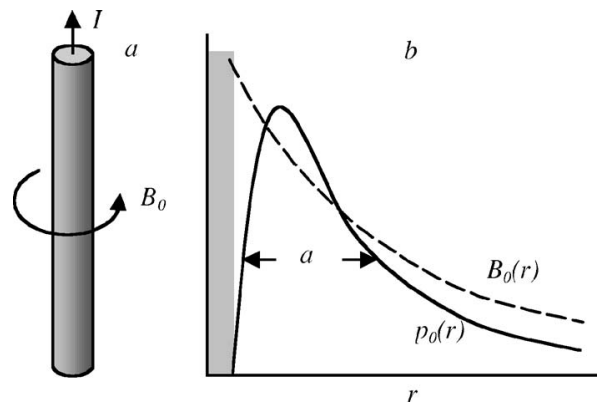

FIG. 1. A low-pressure plasma occupies an area outside a current-carrying rod (panel a). The effect of this plasma on the magnetic field is negligible, so that $B_{0} \sim 1 / r$ (panel $\mathrm{b}$, dashed line). The unperturbed plasma density is uniform, whereas the plasma temperature varies in the radial direction. This creates the pressure variation which is a potential source of the flute instability in the zone where $p_{0}^{\prime}<0$.

tion with artificial diffusivity) and the temperature $T_{0}(\boldsymbol{r})$ varying in the radial direction. The unperturbed pressure $p_{0}(\boldsymbol{r})=2 \rho_{0} T_{0}(\boldsymbol{r}) / m_{i}$ [Fig. 2(b)] varies in the radial direction and serves as a drive for the flute instability. ${ }^{5}$ This geometry exactly corresponds to that of a hard-core pinch $^{6,7}$ and is similar to that of the levitated dipole experiment ${ }^{8,9}$ (where the current-carrying rod forms a loop).

We linearize the set of Eq. (1) and seek perturbations with the $\exp (\Gamma t)$ dependence on time. Denoting the perturbations by the prefix " $\delta$ " and introducing the displacement vector $\boldsymbol{\xi} \equiv \delta \mathbf{v} / \Gamma$, one obtains from the set (1):

$$
\begin{aligned}
& \Gamma^{2} \rho_{0} \boldsymbol{\xi}=-\nabla \delta p+\frac{1}{c} \delta \boldsymbol{j}_{\perp} \times \boldsymbol{B}_{0}, \\
& \delta \rho+\rho_{0} \nabla \cdot \boldsymbol{\xi}=\frac{D}{\Gamma} \nabla^{2} \delta \rho, \\
& \frac{3}{2} \delta T+\frac{3}{2} \boldsymbol{\xi} \cdot \nabla T_{0}+T_{0} \nabla \cdot \boldsymbol{\xi}=0, \\
& 0=\nabla \times \boldsymbol{\xi} \times \mathbf{B}_{0},
\end{aligned}
$$

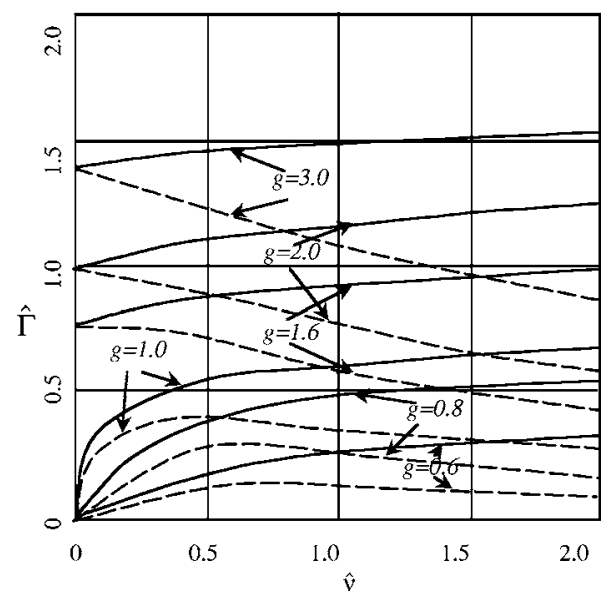

FIG. 2. Dimensionless growth rates for various values of the dimensionless gradient $g$. The solid curves correspond to the zero viscosity. The dashed curves correspond to the case where kinematic viscosity $\eta / \rho_{0}$ is equal to the artificial diffusion coefficient $D$. 


$$
\delta p=p_{0}\left(\frac{\delta \rho}{\rho_{0}}+\frac{\delta T}{T_{0}}\right) .
$$

We have neglected the magnetic field perturbation because, in the pressure-driven modes in a $\beta \ll 1$ plasma, it is of order of $\beta$ compared to the retained terms.

Flute perturbations in the geometry of Fig. 1(a) are axisymmetric perturbations with the azimuthal component of the displacement vector $\boldsymbol{\xi}$ equal to zero. Equation (3d) has then a solution: $\boldsymbol{\xi} \times \boldsymbol{B}_{0}=\nabla \psi$, where $\psi$ is a function independent on the azimuth. From this equation one gets $\xi_{r}$ $=\left(1 / B_{0}\right)(\partial \psi / \partial z), \nabla \cdot \xi=-2 \xi_{r} B_{0}^{\prime} / B_{0}$ where the prime denotes the differentiation over the radius. Substituting this result into Eq. (3c), one finds that

$$
\delta T=-\xi_{r}\left(T_{0}^{\prime}-\frac{4 B_{0}^{\prime}}{3 B_{0}} T_{0}\right),
$$

whereas Eq. (3b) yields

$$
\delta \rho-\frac{D}{\Gamma} \nabla^{2} \delta \rho=\rho_{0} \frac{2 \xi_{r} B_{0}^{\prime}}{B_{0}} .
$$

Expressing $\delta j_{\perp}$ from Eq. (3a) and using the charge neutrality condition $\nabla \cdot \delta \boldsymbol{j} \equiv \nabla \cdot \delta \boldsymbol{j}_{\perp}=0$, one finds that

$$
-\Gamma^{2} \rho_{0}\left(\frac{\partial^{2} \psi}{\partial z^{2}}+\frac{1}{r} \frac{\partial}{\partial r} r \frac{\partial \psi}{\partial r}+\frac{2}{r} \frac{\partial \psi}{\partial r}\right)=2 \frac{\partial B_{0}}{\partial r} \frac{\partial \delta p}{\partial z} .
$$
bations,

At this point, we make an assumption that, in the pertur-

$$
\partial / \partial z \gg \partial / \partial r \gg\left|p_{0}^{\prime} / p_{0}\right| .
$$

It is known from the theory of the flute instability that this condition corresponds to the fastest growing modes. ${ }^{5,10} \mathrm{Un}$ der condition (7), for perturbations of the $\exp \left(i k_{z} z\right)$ type, Eq. (6), together with equation $\xi_{r}=\left(1 / B_{0}\right)(\partial \psi / \partial z)$, yield

$$
-\Gamma^{2} \rho_{0} \xi_{r}=\frac{2}{B_{0}} \frac{\partial B_{0}}{\partial r} \delta p
$$

whereas Eqs. (3e), (4), and (5) yield

$$
\frac{\delta p}{p_{0}}=\xi_{\imath}\left\lfloor-\frac{p_{0}^{\prime}}{p_{0}}+\frac{2 B_{0}^{\prime}}{B_{0}}\left(\frac{2}{3}+\frac{\Gamma}{\Gamma+\nu}\right)\right\rfloor,
$$

where $\nu \equiv k_{z}^{2} D$. Finally, from Eqs. (8) and (9) one obtains the dispersion relation:

$$
\Gamma^{2}=-\frac{2 B_{0}^{\prime}}{B_{0}} \frac{p_{0}}{\rho_{0}}\left\lfloor-\frac{p_{0}^{\prime}}{p_{0}}+\frac{2 B_{0}^{\prime}}{B_{0}}\left(\frac{2}{3}+\frac{\Gamma}{\Gamma+\nu}\right)\right] .
$$

In the case of a zero diffusion coefficient (or, equivalently, the case of a zero $\nu$ ), the dispersion relation acquires a standard form for the flute instability of a gas with $\gamma=5 / 3$. The stability criterion can in this case be presented as

$$
p_{0}^{\prime} U^{\prime}+\frac{\gamma p_{0}}{U} U^{\prime 2}>0
$$

where $U$ is specific volume of the flux tube, $U=\oint d l / B_{0}$ $=(\pi c / I) r^{2}$ and $I$ is the current through the rod [Fig. 1(a)].

In the opposite limit of a very large diffusion coefficient (i.e., at $\nu \rightarrow \infty$ ), one again recovers the stability criterion (11), although with a different $\gamma, \gamma=2 / 3$. In other words, the stability criterion becomes more restrictive. Of course, $\gamma$ cannot be treated in the latter case as a normal adiabatic index, which must be greater than one; in our case, it is just a parameter of the solution.

As we have already mentioned, dispersion relation (10) is a local dispersion relation: it describes the evolution of perturbations with a length scale smaller than the gradient scale length $\left|p_{0} / p_{0}^{\prime}\right|$. Assume that we are studying the instability in the vicinity of the surface $r=a$. We introduce the dimensionless growth rate $\hat{\Gamma}$, the dimensionless pressure gradient $g$, and the dimensionless $\nu: \hat{\Gamma}=\Gamma a / 2 s ; g=-3 a p_{0}^{\prime} / 10 p_{0}$; $\hat{\nu}=\nu a / 2 s$, where $s \equiv \sqrt{5 p_{0} / 3 \rho_{0}}$ is the adiabatic sound speed. Numerical coefficients here are chosen in such a way as to make $g=1$ correspond to the critical gradient in the "ideal" case $(D=0)$. Note that this instability is very fast: for $\hat{\Gamma}=1$, the dimensional e-folding time is $a / 2 s$.

The dimensionless dispersion relation reads as

$$
\hat{\Gamma}^{2}=g-1+\frac{0.6 \hat{v}}{\hat{\Gamma}+\hat{\nu}} .
$$

One can show that, at $g>0.4$, this equation has one unstable root with a purely exponential growth. The other two roots are stable. For $g<0.4$ all roots are stable.

The unstable domain can be split in two subdomains, $0.4<g<1$ and $g>1$, with the growth rate behaving quite differently (Fig. 2). In the first subdomain, the growth rate is zero at $\nu=0$ (i.e., at zero diffusivity). At small but finite values of $\nu$, the growth rate is proportional to $\nu$ and can be approximately represented as $\hat{\Gamma}=\hat{\nu}(g-0.4) /(1-g)$ (this expression is valid for $g$ not too close to the standard instability boundary $g=1$ ). The growth rate is proportional to the diffusivity - a feature of what would normally be called a "negative energy mode." In our case, as we have $g<1$ (i.e., we are below the threshold for the standard flute instability), the potential-energy perturbation is actually positive. The occurrence of the analog of a negative energy mode is related here to the aforementioned fact that the artificial diffusivity cannot be easily interpreted in terms of its effect on the energy conservation [cf. Eq. (2)].

Note that the instability at $0.4<g<1$ is present even at very small values of the diffusivity. Asymptotically, at high $\nu$, the unstable solution of Eq. (12) reaches the level characteristic of the flute instability with $\gamma=2 / 3, \hat{\Gamma} \approx \sqrt{g-0.4}$.

At $g>1$ the situation is quite different (Fig. 2). First, the growth rate is positive even at $\nu=0$; second, if $g$ exceeds the critical value $(g=1)$ by a factor of 2 or more, the growth rate becomes relatively independent of the artificial diffusion. This is a direct consequence of the structure of Eq. (12): the second term varies in the limited interval between 0 and 0.6. Thus, its effect on the growth rate at $g \gg 1$ becomes small (because the first term is large).

Consider now the case where a resistive term is included in Eq. (1d) [see a comment following Eq. (2)] and the artificial diffusivity is omitted. One can show that, in the limit defined by Eq. (7), the resistive term enters the problem via the inverse diffusion time over the scale $1 / k_{z}, \nu_{r}=2 D_{r} k_{z}^{2} / 3$, 
where $D_{r}=c^{2} p_{0} / B_{0}^{2} \sigma$ can be called the resistive diffusion coefficient. In the dimensionless variables, the dispersion relation acquires the form $\hat{\Gamma}\left(\hat{\Gamma}+\hat{\nu}_{r}\right)=g-1$, where $\hat{\nu}_{r}=\nu_{r} a / 2 s$. The resistive diffusion term may become important for anomalously low conductivity $\sigma$. In terms of its effect on the instability, it gives rise to an expected reduction of the growth rate but does not lower the stability threshold as the artificial diffusivity $D$ does. Therefore, it would be incorrect to identify the artificial diffusivity $D$ in the continuity equation Eq. (1b) with the resistive diffusivity $D_{r}$ (even if it is anomalous).

Now we briefly consider the effect of viscous dissipation, which will be accounted for together with the artificial diffusivity. We include it by adding a term $\eta \nabla^{2} \mathbf{v}$ to the righthand side of Eq. (1a). In the limit defined by Eq. (7), the following dimensionless dispersion relation holds:

$$
\hat{\Gamma}\left(\hat{\Gamma}+\hat{\nu}_{\text {visc }}\right)=g-1+\frac{0.6 \hat{\nu}}{\hat{\Gamma}+\hat{\nu}},
$$

where $\hat{\nu}_{\text {visc }}=\nu_{\text {visc }} a / 2 s, \nu_{\text {visc }}=\eta k_{z}^{2} / \rho_{0}$.

At a zero artificial diffusivity $\hat{\nu}=0$, the viscous term does not affect the plasma stability boundary, which remains $g$ $=1$. Viscosity leads just to a reduction of the growth rate, and modes mimicking the negative energy modes are absent. However, when the artificial diffusivity is turned on, the stability threshold becomes lower and the modes with a growth rate proportional to $\hat{\nu}$ appear.

As an illustration of the effect of viscosity on the growth rate, dashed lines on Fig. 2 show the growth rate for the case where $\nu_{\text {visc }}=\nu$ (in other words, for quite high kinematic viscosity $\eta / \rho_{0}$, equal to the artificial diffusivity $D$ ). One sees that the growth rates become somewhat smaller, but no dramatic changes occur compared to the zero viscosity case.

Based on these results, we come to the following conclusions regarding the effect of the artificial diffusivity on the pressure-driven modes. The effect is unimportant if

$$
\Gamma \gg D / \chi^{2},
$$

where $\chi$ is a characteristic scale length of perturbations. If this condition is violated, two outcomes are possible. If the contribution of the gas compressibility [the terms proportional to $\gamma$, Eq. (11)] to the growth rate is small, the growth rate remains insensitive to the artificial diffusivity (despite the fact that $D$ is "large"). Conversely, if the condition (14) is violated and, at the same time, the dependence of the growth rate on $\gamma$ is substantial, the effect of the diffusivity on the instability becomes significant, leading to the broadening of the instability range and introducing modes behaving like negative energy modes. Adding viscous terms does not affect these conclusions, at least in a qualitative way. The anomalous diffusion caused by resistive effects also does not change the stability limit; it only somewhat decreases the growth rate.

Our results may serve as guidance in assessing the effect of a finite $D$ on pressure-driven modes in numerical simulations. They may also provide some insights into the effect of a large $D$ on other modes, e.g., current-driven modes. Here it is reasonable to assume that the role of a large $D$ will be insignificant if the modes do not depend substantially on the plasma compressibility, which is true, in particular, for the paraxial modes. ${ }^{11}$

We thank Dalton Schnack for his assistance with the literature on numerical MHD and for encouragement.

Work performed for the U.S. Department of Energy by the University of California Lawrence Livermore National Laboratory, Contract No. W-7405-Eng-48.

${ }^{1}$ C. R. Sovinec, B. I. Cohen, G. A. Cone, E. B. Hooper, and H. S. McLean, Phys. Rev. Lett. 94, 035003 (2005).

${ }^{2}$ B. I. Cohen, E. B. Hooper, R. H. Cohen, D. N. Hill, H. S. McLean, R. D. Wood, S. Woodruff, C. R. Sovinec, and G. A. Cone, Phys. Plasmas 12, 056106 (2005).

${ }^{3}$ C. R. Sovinec, A. H. Glasser, T. A. Gianakon, D. C. Barnes, R. A. Nebel, S. E. Kruger, D. D. Schnack, S. J. Plimpton, A. Tarditi, M. S. Chu, and the NIMROD Team, J. Comput. Phys. 195, 355 (2004).

${ }^{4}$ D. D. Schnack, Z. Mikic, D. S. Harned, E. J. Caramana, and D. C. Barnes, J. Comput. Phys. 70, 330 (1987).

${ }^{5}$ M. N. Rosenbluth and C. L. Longmire, Ann. Phys. 1, 120 (1957).

${ }^{6}$ R. E. Siemon, B. S. Bauer, A. Esaulov, V. Makhin, R. Presura, V. I. Sotnikov, D. D. Ryutov, I. R. Lindemuth, P. T. Sheehey, and R. C. Kirkpatrick, Bull. Am. Phys. Soc. 48, 339 (2003).

${ }^{7}$ V. Makhin, R. E. Siemon, B. S. Bauer, A. Esaulov, I. R. Lindemuth, D. D. Ryutov, P. T. Sheehey, and V. I. Sotnikov, Phys. Plasmas 12, 042312 (2005).

${ }^{8}$ J. Kesner and M. Mauel, Plasma Phys. Rep. 23, 742 (1997).

${ }^{9}$ J. Kesner, A. N. Simakov, D. T. Garnier, P. J. Catto, R. J. Hastie, S. I. Krasheninnikov, M. E. Mauel, T. S. Pedersen, and J. J. Ramos, Nucl. Fusion 41, 301 (2001).

${ }^{10}$ B. B. Kadomtsev, in Reviews of Plasma Physics, edited by M. A. Leontovich (Consultants Bureau, New York, 1966), Vol. 2, p. 153.

${ }^{11}$ J. P. Freidberg and L. D. Pearlstein, Phys. Fluids 21, 1207 (1978). 